\title{
NOTE SULLA SINTASSI DELL'ACCUSATIVO PREPOSIZIONALE IN ITALIANO
}

\section{PREMESSA}

In un breve quanto limpido paragrafo della sua Grammatica storica dell'italiano ( $\$ 553$ dell'ed. 1972, § 436 nell'ed. 1980), Pavao Tekavčić descriveva l'accusativo preposizionale dei dialetti italiani centromeridionali, accennandone $i$ confini geografici e collegandolo, molto correttamente, con il fenomeno analogo delle lingue iberoromanze e del rumeno. Ne forniva anche, nella scia della migliore tradizione di pensiero sull'argomento, una convincente spiegazione funzionale: la preposizione funge da segnacaso esplicito per quegli oggetti che, avendo referente umano, sarebbero interpretabili come soggetti se non fossero marcati (cfr. già Diez $1882^{5}$, III., pp. 835 s.; oggi Bossong 1985 e 1986 per una panoramica tipologica, e 1988 per le lingue romanze). ${ }^{1}$

Vorremmo in questa sede tornare sull'argomento, focalizzando l'attenzione sull'accusativo preposizionale non tanto nei dialetti italiani, quanto nell'italiano, lingua romanza che, almeno secondo la manualistica el'opinione corrente, non avrebbe accusativo preposizionale (assieme al francese: cfr. per es. Körner 1987).

L'italiano standard in realtà conosce l'accusativo preposizionale (d'ora in poi acc.prep.), benché in un uso ristretto ad una gamma piuttosto limitata di contesti, finora relativamente poco studiata (ma si cfr. ora Benincà 1986, Nocentini 1985 e 1987, Renzi 1988, pp. 155-156, Zamboni 1989, ed anche Berretta 1989b, 1990a e 1990b). Non sarà quindi inopportuno, anche a semplici fini descrittivi, l'esame di alcuni dati empirici sull'argomento.

Riferiremo, nei paragrafi che seguono, sui risultati dell'analisi morfosintattica di cento occorrenze ${ }^{2}$ di acc.prep. in italiano parlato e scritto, tutte riportate qui in

1 La bibliografia sull'accusativo preposizionale è vastissima, e non è questa la sede in cui tentarne una rassegna: rinvio per questo a Zamboni 1989 , al cui elenco tengo ad aggiungere solo la tesi inedita di Lore Terracini, che ho potuto consultare grazie alla cortesia dell'Autrice (Terracini 1950, la cui seconda parte è dedicata interamente all'accusativo preposizionale nel Libro de buen amor, con un'ampia introduzione teorica).

2 Si tratta delle prime cento occorrenze fra quelle che erano state raccolte inizialmente per lavori precedenti sullo stesso tema (Berretta 1989b, 1990a e 1990b) e poi in tempi successivi, per questo contributo. Alcune di esse sono dovute alla cortesia di amici e collaboratori, che le hanno annotate per me: colgo qui l'occasione per ringraziarli di cuore. 
appendice. In particolare vedremo: i tipi di nominali che si trovano marcati con $a$ all'accusativo, la loro natura pronominale o nominale, la persona, il tipo di referenti, ecc. $(\$ 2$.); la loro posizione rispetto al verbo (\$ 3.) e agli altri costituenti della frase, con particolare riferimento al soggetto $(\S 4$.); i verbi o complessi verbali che reggono gli acc.prep. $(\S 5$.); nonché la presenza o assenza di pronomi atoni di ripresa o anticipazione ( $\$ \S 2$. e 5.). Cercheremo anche, nei limiti in cui le ridotte dimensioni del campione lo permettono, di correlare l'una all'altra le variabili osservate.

\section{IL CORPUS}

Prima di passare all'analisi, è necessario spendere qualche parola per una caratterizzazione sociolinguistica del corpus (e quindi del fenomeno ivi indagato), ovvero sulla sua collocazione nella complessa architettura delle varietà dell'italiano.

Il primo punto è la caratterizzazione diatopica: è noto infatti che varietà regionali di italiano, meridionali e insulari, per effetto dei dialetti retrostanti, hanno acc.prep. in una gamma d'usi relativamente ampia (Rohlfs 1954, § 632, 1971, 1973; Elia 1980; Leone 1982, p. 137; Loi Corvetto 1983, pp. 112-114; Serianni 1988, p. 81). Ma la varietà che in questa sede intendiamo esaminare è l'italiano, se non standard in senso stretto, almeno «dell'uso medio» (secondo la felice denominazione di Sabatini 1985; cfr. anche la nozione di «neo-standard» in Berruto 1987), non marcato regionalmente. Abbiamo quindi scartato, soprattutto per gli esempi di parlato, dati di parlanti provenienti dall'Italia centro meridionale e dalle isole, indipendentemente dalla loro istruzione.

Gli esempi orali pertanto sono stati tratti dal parlato informale (talvolta anche semiformale o formale) di persone provenienti dall'Italia settentrionale (in maggioranza si tratta di piemontesi e lombardi), ${ }^{3}$ zona nella quale i dialetti sembrano ignorare l'acc.prep.: dovremmo così essere sicuri che i fenomeni osservati non costituiscano rispecchiamenti di strutture dialettali. Globalmente gli esempi di parlato sono 78 sulle 100 occorrenze schedate.

Gli esempi scritti (22 su 100) provengono invece da giornali e riviste, e in pochi casi da testi letterari (la fonte viene comunque citata ogni volta). Per lo più si tratta di frammenti di interviste o battute di dialogo, dunque di riproduzioni/ricostruzioni più o meno fedeli della lingua parlata, oppure di scritto volutamente informale (per esempio, in forma epistolare). Ovviamente negli esempi di questa parte del corpus la provenienza regionale dello scrivente è meno controllabile, anche per il sovrapporsi di più 'mani' (fonte della notizia, autore dell'articolo, redattore): tuttavia lo statuto di lingua scritta ci pone di per sé in un ambito sovraregionale e in una varietà, se non proprio standard, certo 'standard media'.

3 Si tratta per lo più di persone colte (spesso docenti universitari, studenti pure universitari, insegnanti ecc.); solo in pochi casi i parlanti sono semicolti, o molto giovani. Talvolta non ho informazioni sicure sul grado di istruzione del parlante. 
Per quanto riguarda la caratterizzazione diastratica, $i$ dati provengono, nella stragrande maggioranza, da parlanti colti o molto colti (per esempio vi sono sovrarappresentati docenti universitari). Abbiamo voluto evitare il ricorso a materiali di italiano popolare, sia parlato che scritto e indipendentemente dalla provenienza regionale dei parlanti/scriventi, perché ci era noto da lavori precedenti che in varietà diastratiche basse anche settentrionali le regole d'uso dell'acc.prep. tendono ad essere più 'larghe' che nell'italiano colto, e costituiscono anzi un continuum con le varietà centrali e meridionali (Berretta 1989b, pp. 23-24 e 1990a, 32-33). In diastratia il nostro corpus è da considerare quindi non marcato o, volendo, marcato come medio-alto. E' invece basso o medio basso in diafasia, per l'ampia presenza di dati dal parlato colloquiale e dallo scritto che riproduce o imita il parlato.

\section{PRONOMI E NOMI}

Nella descrizione che P. Benincà (in Renzi 1988) ha dato dell'acc.prep. in italiano standard, la prima restrizione d'uso riguarda la natura dei costituenti di frase che possono essere marcati con $a$ quando siano nel ruolo di oggetti: deve trattarsi di pronomi deittici, preferibilmente singolari. In altri termini, deve trattarsi dei tipi di nominali di grado più alto nella gerarchia sia di animatezza per caratteristiche referenziali inerenti, sia di definitezza, e come tali più inclini - se aventi ruolo di oggetto diretto - ad essere marcati in modo esplicito in sistemi a marcatura differenziale dell'oggetto (Silverstein 1976, Moravcsik 1978, Comrie 1979, Lazard 1984, Bossong 1988).

L'andamento dei nostri dati conferma questa restrizione, con poche eccezioni: abbiamo infatti $91 / 100$ pronomi di prima e seconda persona, di cui 76 singolari e 15 plurali (cfr. tavola 1.). Vi è una preferenza molto chiara per la prima persona, sia al singolare (59 occorrenze, più di metà del totale: cfr. in appendice 9 , a me colpi moltissimo...; 16, a me mi conoscono; 18, non mi convince $A$ ME; 64, a me mi hanno pagato; ecc.) che al plurale (13 su un totale di 18 plurali; cfr. 50 , a noi non c'hanno informato; 57, lasciateci salire a noi; ecc.). ${ }^{4}$ La seconda persona segue la prima per numero di occorrenze al singolare (17: cfr. 25 , se a te non ti disturba; 65 , a te ti pa$g a$; ecc.), ma non al plurale, dove è di poco superata dalla terza: in numeri assoluti il dato pare irrilevante, ma è coerente con la generale scarsa frequenza statistica della II. plurale (cfr. i due soli esempi: 41 , ma a voi fa ridere uno che dice [...] e 4, vi aspetto più tardi + a tutt'e due).

4 Qui come nell'appendice, nelle trascrizioni di parlato sono usati i seguenti segni speciali: + per pausa breve/brevissima; $++\mathrm{e}+++$ per pause, più significative; / per autointerruzione; maiuscolo per enfasi; un trattino non spaziato in fine di parola per intonazione sospensiva. La virgola indica cesura intonativa; puntini di omissione, glosse esplicative e annotazioni sono fra parentesi quadre. Pronomi di III. persona usati come allocutivi di cortesia (di II.) sono segnalati con la maiuscola iniziale. 
Tav. 1. Persona e numero

\begin{tabular}{llrcr} 
& I. & II. & III. & Tot. \\
singolare & 59 & 17 & 6 & 82 \\
plurale & 13 & 2 & 3 & 18 \\
Tot. & 72 & 19 & 9 & 100 \\
\hline
\end{tabular}

Alle persone deittiche troviamo ovviamente pronomi personali tonici; vi sono solo alcuni nominali pieni aggiunti come apposizioni al pronome di 1 . plurale $(56, a$ noi bianchi; 61 , a noi nordafricani, e 72, a noi comuni mortali) e un caso in cui non è un pronome bensi un quantificatore ( $a$ tutt'e due, citato qui sopra) che rinvia ad una II. plurale.

La presenza di nominali pieni è invece importante alla III. persona, dove su 9 occorrenze troviamo solo due pronomi personali (3, a loro le aspettava Adone; 29, a loro disturba), tre altri pronomi, tutti con referenti umani (due interrogativi: $a$ chi, 7 e 45; un dimostrativo: 98 , non lo vedi più a quello) e quattro nomi. Di questi ultimi solo uno è un nome proprio di persona, il caso che più ci si aspettava per combinazione di tratti referenziali inerenti (animato, umano) con definitezza e specificità (76, a Cerami preoccupa Viterbo); poi abbiamo un nome comune con referente umano, al plurale (33, ai bambini l'idea non disturba) e ancora un nome comune con referente non umano ma animato $(8$, al gatto io lo coccolo - dove si noti l'implicito possessivo di I. singolare, che aumenta il grado di specificità del nominale). Una occorrenza infine devia dalla tendenza generale, non per tratti referenziali (il referente resta animato e umano) ma per l'indefinitezza: è l'esempio (scritto) riportato in 10 , ad un linguista possono colpire particolarmente [...] frasi del seguente tenore. ${ }^{5}$

La comparsa di pronomi di terza persona e di nomi propri all'accusativo preceduti da $a$ era già stata segnalata da Benincà (1986, pp. 74-75 e in Renzi 1988, pp. 133-135) nel caso in cui il predicato reggente sia un verbo psicologico, per esempio in frasi come $A$ Giorgio, questi argomenti non l'hanno convinto, sempre con la restrizione che l'oggetto sia dislocato a sinistra, cioè sia in posizione preverbale, separato dal resto della frase almeno da una pausa virtuale, e abbia ripresa con un clitico (cfr. qui § 5.). Ma i nostri quattro nominali pieni non sono tutti nomi propri, non sono tutti ripresi da clitici, e almeno uno è retto da un verbo che non sembra potersi classificare come psicologico, coccolare.

Nell'insieme quindi i nostri dati confermano le restrizioni d'uso citate all'inizio per quanto riguarda la natura degli oggetti marcati con preposizione, ma mostrano che tali restrizioni non sono categoriche. Come è risultato dalle ricerche sulla marca-

5 Si noti peraltro che nell'esempio citato, a causa della posizione preverbale, non è neppure possibile la cancellazione di $a$; l'unica soluzione standard che permetta di mantenere un linguista a tema e frasi del seguente tenore a rema sarebbe una frase passiva. 
tura differenziale dell'oggetto in tipologia, gli oggetti si distribuiscono in un continuum che ha ad un polo elementi altamente specifici, pronomi deittici singolari di prima persona, e al capo opposto oggetti prototipici, nomi con referenti inanimati non specifici (in particolare nomi di massa). Le occorrenze di acc.prep. si concentrano sul polo alto, e si distribuiscono poi in misura decrescente sui gradini inferiori. La soglia minima individuata si colloca alla III. persona plurale, con nominali aventi referenti umani o almeno animati, di regola definiti ma con almeno una eccezione, il citato ad un linguista (si noti anche il caso di ai bambini, che è formalmente definito ma non specifico). Per quanto può valere un singolo esempio - cui però vanno aggiunti i due casi col pronome interrogativo $a$ chi -, parrebbe che fra i due tratti in potenziale concorrenza, /umano/ e /definito/ (cfr. Comrie 1979, Bossong 1988), l'italiano tenda a far prevalere il primo, il che sarebbe coerente con quanto si sa sull'uso dell'acc.prep. nei dialetti italiani meridionali e insulari: pensiamo per es. all'acc.prep. coll'indefinito 'nessuno' o con nominali indefiniti: siciliano num bògghiu a nnuḍdu cu mmia «non voglio nessuno con me», a nu scarparu no vogghiu pi mmarito «un calzolaio non lo voglio per marito», e simili (cfr. Rohlfs 1971 e 1973).

\section{POSIZIONE NELLA FRASE}

Secondo la linea di pensiero citata all'inizio, cui anche Tekavčić aderisce, per la quale l'acc.prep. è una forma di marca di caso che esplicita un ruolo sintattico altrimenti ambiguo, vi è una correlazione non solo fra presenza della marca e caratteristiche referenziali non canoniche dell'oggetto, ma anche fra presenza della marca e posizione non canonica dell'oggetto: l'acc.prep. sarebbe più probabile per oggetti in posizione preverbale, la posizione caratteristica del tema o topic di frase. In italiano, sempre secondo Renzi 1988, tale posizione sarebbe l'unica in cui sia possibile acc.prep., con l'ulteriore restrizione che l'oggetto deve essere dislocato a sinistra, cioè - nella descrizione che la Grande grammatica dà delle dislocazioni - separato dal nucleo della frase almeno da una «pausa virtuale» e ivi ripreso da un pronome atono. 6

I nostri dati confermano la preferenza per la posizione preverbale, ma mostrano non poche eccezioni (cfr. tavola 2): come vedremo, nell'insieme i nostri risultati si discostano dalla descrizione canonica dell'acc.prep. italiano più per la sintassi che per le caratteristiche degli oggetti, di cui s'è detto al § precedente.

Tav. 2. Posizione pre/postverbale e ripresa con clitico

\begin{tabular}{lcccc} 
& prev. & postv. & Tot. & (indecid.) \\
con ripresa & 36 & 15 & 51 & \\
senza ripresa & 38 & 3 & 41 & \\
Tot. & 74 & 18 & 92 & (8) \\
\hline
\end{tabular}


In totale, gli acc.prep. in posizione preverbale sono 74 (ess.: 22, a me danneggia...; 58, finora a noi ci lasciavano stare) mentre 18 occorrono in posizione postverbale (ess.: 46, mi hanno fucilato $A$ ME?; 90, ho pensato quanto avrebbe stancato $A$ $M E$ quel viaggio). Il residuo è dato da 8 casi non decidibili perché ellittici di verbo: si tratta di risposte brevi o frammenti aggiunti, in cui il verbo reggente è recuperato anaforicamente dalla battuta o parte di battuta precedente (cfr. per es. in 45, A: ed è li che ti frego B: $A$ CHI?; in 15, non è che ci conoscano tanto + a te magari si). ${ }^{7}$ In percentuale sul totale delle occorrenze 'decidibili' (92) abbiamo 80,4\% di casi di posizione preverbale e $19,6 \%$ di casi di posizione postverbale: la seconda percentuale, come si vede, è bassa ma non marginale.

Lasciando per un momento il livello sintattico vero e proprio per passare alla dimensione testuale, si noti, nella misura in cui la brevità degli esempi riportati lo consente, che spesso l'acc.prep. occorre all'inizio di un turno conversazionale: si tratta chiaramente di inserimenti di un nuovo tema di frase (eventualmente con lieve valore contrastivo rispetto a quanto precede), all'interno di un argomento di discorso che permane. Così ad esempio in (64) il frammento a me mi hanno pagato segue una battuta relativa ad altra persona che invece non era stata pagata; in (81) per fortuna a me la dieta non rattrista l'interlocutore aveva appena detto di essere di malumore perché a dieta, e così via (cfr. anche 22, 59, 71, e altri, ivi comprese le risposte brevi ellittiche di verbo, come 26: A: vi disturbo se faccio fotocopie mentre voi parlate? B: a me, no). Da questo punto di vista non è però direttamente l'acc.prep. ad essere correlato alla lieve discontinuità testuale: lo sono piuttosto $\mathrm{i}$ pronomi tonici, in opposizione agli atoni (si confronti l'effetto di discontinuità di $a$ me la dieta non rattrista con l'effetto invece di continuità del parallelo la dieta non mi rattrista); a loro volta poi i pronomi tonici oggetto sono correlati con l'acc.prep., che invece con gli atoni ovviamente non è possibile.

Ma torniamo all'analisi sintattica, cui il nostro materiale meglio si presta. Come s'è detto sopra, nel caso giudicato normale ci si aspetta che l'acc.prep. sia richiamato da un pronome atono. Effettivamente la ripresa con un pronome atono è più spesso presente (51 casi; per es. 65-66, a te ti paga e a me pure mi paga) che assente (41 casi; es. 20, a me non convince), tuttavia la differenza è meno significativa rispetto a quella relativa alla posizione: facendo il rapporto di nuovo sul totale di $\mathbf{9 2}$ occorrenze (se vi è ellissi di verbo, anche la presenza o assenza del clitico ad esso legato non è decidibile) abbiamo 55,4\% di acc.prep. con ripresa clitica e 44,6\% senza. Come vedremo meglio più avanti ( $\$ 5$.), la presenza/assenza del pronome atono

6 Secondo tale interpretazione tutti gli elementi dislocati, ivi compresi gli acc.prep., non farebbero quindi parte della frase vera e propria, ma semmai dell'enunciato. In altra sede ho cercato di argomentare come, invece, i nominali dislocati siano ormai da considerare integrati nella struttura frasale (Berretta 1989a e in stampa, § 4.2.). Ritorneremo sull'argomento, limitatamente agli acc.prep., qui nel $\S 5$.

7 Gli esempi classificati come indecidibili sono i numeri: $13,15,26,45,62,82,86,96$. In 13 si è considerato a me no come una profrase autonoma (in questo caso una autorisposta), anche se il parlante poi prosegue con non mi conforta. 
è correlata al tipo di verbo reggente, con netta preferenza dei verbi psicologici e dei complessi verbali causativi per l'assenza del clitico.

Di qualche interesse è l'incrocio dei dati relativi alla posizione con quelli di presenza o assenza del clitico: acc.prep. preverbali occorrono lievemente più spesso senza clitico che con ( 38 casi su $74,51,35 \%$, senza clitico, contro $36 / 74,48,65 \%$, con clitico), mentre acc.prep. postverbali preferiscono di gran lunga la ripresa, $o$ anticipazione se si preferisce, col pronome atono (15 casi su $18,83,3 \%$, contro $3 / 18,16,7 \%$ ). I tre tipi più frequenti di acc.prep. per tratti sintattici sarebbero quindi, nell'ordine: (i) preverbale senza ripresa, come in a me diverte (ess. 34 e 35); (ii) preverbale con ripresa come in a me nessuno mi protegge (es. 79); e (iii) postverbale con ripresa, come in non t'ho visto a te (es. 100).

La maggiore frequenza di riprese con clitico nei casi di posizione postverbale è un indizio della relativa maggiore marcatezza dell'acc.prep. in questa posizione, che sembra meno integrato nella frase rispetto alla posizione preverbale. Lo stesso emerge da brevi pause che separano la frase dall'acc.prep. (es. 4, vi aspetto più tardi $+a$ tutt'e due), da enfasi contrastiva sull'acc.prep. stesso (ess. 18, non mi convince $A$ $M E, 46$, mi hanno fucilato $A M E$ ?, 90, ho pensato quanto avrebbe stancato $A M E$ quel viaggio, e altri), ${ }^{8}$ o da un avverbio che focalizza l'oggetto (come in primo luogo in 19: non mi convince, $A M E$ in primo luogo, o anche in 70: un'altra volta ci portate anche a noi). Questi stessi indizi mostrano che gli acc.prep. postverbali tendono ad avere statuto di focus, come quelli ellittici di verbi introdotti da anche/neanche. E' importante anche la maggiore marcatezza sociolinguistica della posizione postverbale, che compare più spesso in frammenti prodotti da parlanti semicolti (ess. 1 , e te tu m'accompagni anche a me, 68 , ci picchiava a noi?!, 83, 84) o studenti (ess. $98,100)$ o ragazzi molto giovani (ess. 51,70 ), o, ancora, compare in varietà speciali, quali baby talk (es. 14, io ti conosco, a te!, detto ad una bambina piccola) e pet talk (es. 47, $t i$ piacerebbe se io ti graffiassi così $A T E$ ?, detto al gatto di casa). Viceversa, tutte le occorrenze di acc.prep. in testi scritti, $22 \mathrm{su} 22$, sono preverbali.

\section{ORDINE DEI COSTITUENTI: OGGETTI E SOGGETTI}

Una volta verificata la preferenza dell'acc.prep. per la posizione preverbale, possiamo cercare di approfondire il suo comportamento sintattico esaminandone la posizione nei confronti del soggetto: se è vero che l'acc.prep. tende ad essere topic frasale, dovrebbe anche tendere a precedere il soggetto. ${ }^{9}$

8 Per esempio chiaramente contrastivi sono anche gli ess. (57) e (99), dove pure non c'è particolare enfasi nella pronuncia: in ma intanto lasciateci salire a noi il 'noi' è riferito a "passeggeri normali», in contrasto con un gruppo di tifosi che la polizia non lascia salire sul treno; in vorrei vederti a te fare otto ore di allenamento al giorno abbiamo quasi una formula fissa di contrasto.

9 In generale tratteremo assieme le occorrenze di acc. prep. in frasi principali (che sono la maggioranza) e in dipendenti (per es. relative - ess. 29 e 19 -, completive - es. 23 -, causali - es. 49 -), ma metteremo in evidenza $i$ casi in cui la struttura della dipendente abbia influenza sull'ordine dei costituenti. Lo stesso vale per le occorrenze in frasi assertive o invece interrogative. 
Sempre tenendo come riferimento le 92 frasi con verbo espresso, troviamo in esse vari ordini dei costituenti maggiori, distribuiti come segue (e come riassunto alla tavola 3.). Nella metà dei casi, 46 , non c'è soggetto espresso, e abbiamo quindi OV (34) o VO (12) a seconda della posizione dell'acc.prep., pre- o postverbale.

Tav. 3. Ordine dei costituenti maggiori (con $\mathrm{O}=$ acc.prep)

\begin{tabular}{lrrrrrrr} 
& OV & VO & OVS & OSV & VOS & SOV & SVO \\
prev. & 34 & 0 & 24 & 12 & 0 & 4 & 0 \\
post. & 0 & 12 & 0 & 0 & 1 & 0 & 5 \\
Tot. & $46(\varnothing S)$ & & \multicolumn{3}{c}{$37(\mathrm{O}>\mathrm{S})$} & & $9(\mathrm{~S}>\mathrm{O})$ \\
\hline
\end{tabular}

Il soggetto ellittico è molto spesso un elemento anaforico (quindi con carattere di topic discorsivo, non in conflitto col topic frasale): così per es. in (24) a me ha deluso moltissimo, il cui soggetto, la Bretagna, era stato introdotto poco prima (cfr. es. 23), o in (32) io sento un piccolo rumore di fondo [...] a me comunque non mi disturba. ${ }^{10}$ Talvolta è un soggetto generico, soprattutto nel tipo OV, dove la struttura risultante equivarrebbe, in una varietà molto formale, ad un passivo senza agente; cfr. in (59) a me hanno mandato giù per sbaglio, in (61) a noi nordafricani ci metteranno tutti in galera (altri ess. analoghi sono 63, 64, 71, 72, 73, anche l'impersonale 52, a me non mi si inganna). Infine, può trattarsi di un soggetto deittico, come in (4) vi aspetto più tardi + a tutt'e due; (98)-(100) non lo vedi più a quello; vorrei vederti a te fare otto ore di allenamento al giorno; ma non t'ho visto a te. Nei limiti dei piccoli numeri di cui disponiamo, sembra esservi una correlazione fra il tipo VO e l'ellissi di soggetto deittico (cfr. fra gli altri esempi 57, 70 e 84); si vedano però anche altri casi, come (97) a me non mi vedi fare queste cose (ordine OV).

Quando il soggetto è espresso, nella grande maggioranza dei casi, 37 su 46 $(80,4 \%)$, esso segue l'oggetto; solo in 9 casi $(19,6 \%)$ lo precede. La tendenza attesa è quindi confermata, l'ordine $\mathrm{O}>\mathrm{S}$ prevale su $\mathrm{S}>\mathrm{O}$, il che sembra andare contro ad uno degli universali empirici più noti, ${ }^{11}$ ma va invece a sua conferma, perché gli

10 La brevità dei frammenti riportati, dovuta qui a limiti di spazio ma talvolta anche alla annotazione manuale degli esempi, non permette sempre di distinguere i soggetti ellittici di terza persona anaforici dai deittici; altri esempi chiari di anaforici sono in 88, 89 e 95 . Deittico dovrebbe essere invece $94, a$ me non mi teme, riferito ad un animale domestico (presente nella situazione).

11 Mi riferisco al noto universale n.ro 1 di Greenberg 1963, «Nelle frasi dichiarative con soggetto e oggetto nominali, l'ordine dominante è quasi sempre quello in cui il soggetto precede l'oggetto", confermato - sempre in termini di tendenza e non di universale assoluto - da più ricerche empiriche. 
oggetti che stiamo esaminando hanno caratteristiche ben più da soggetti prototipici che da oggetti. ${ }^{12}$

Nell'insieme i dati della tavola 3. mostrano che gli ordini che sono considerati canonici per l'italiano, VO con ellissi del soggetto e soprattutto SVO con soggetto espresso, sono minoritari se non marginali rispetto a ordini non canonici quali OVS ( 24 casi), OSV (12 casi) e altri: ciò conferma globalmente che nelle frasi con acc. prep. i costituenti nominali maggiori, non solo l'oggetto ma anche il soggetto, hanno un comportamento sintattico diverso da quello normale.

La posizione postverbale del soggetto ( 25 occorrenze, di cui 24 con ordine OVS e una con ordine VOS) è dovuta nella maggior parte dei casi a soggetti rematici, talvolta anche 'pesanti' sintatticamente: si vedano esempi come (9) a me colpì moltissimo il fatto che anni fa si scoprisse che [...], (11) a me ha colpito un aspetto in particolare, (22) a me danneggia tantissimo quello della settimana dopo [si parla di scioperi dei ferrovieri], (77) anche a me preoccupa molto il fatto da Lei denunciato, (92) a noi c'ha svegliato il telefono, verso le quattro [convenevoli tra vicini di casa, la mattina del primo dell'anno: si parla dei disturbi della notte], ecc. (cfr. anche ess. 3, $10,23,34,35,41,75,76,78,85,93) .{ }^{13}$ Vi sono però anche posposizioni che paiono dislocazioni a destra del soggetto, aggiunte come per ripensamento; in questi casi il soggetto è noto, e viene esplicitato dopo la frase, da cui è separato da una cesura intonativa (il caso è quindi analogo più all'ellissi di soggetto che alla vera posposizione): cfr. (44) a me m'hanno fermato, la polizia, (49) perché a me m'incastra, quello ai. ${ }^{14}$

E' da notare che nel corpus compaiono anche sei frasi scisse o pseudoscisse; di queste 5 hanno l'oggetto al primo posto, fuori dalla complessa struttura della (pseudo)scissa, in posizione tipica di tema: cfr. (6) a me quello che attrae di più è l'idea [...] o (91) a me è 'sto periodo di caldo che mi ha steso e gli esempi analoghi (2), (5) e (40). Solo in un caso l'oggetto è in posizione interna alla pseudoscissa: (12) quello che a me colpisce è [...]. Per semplicità, nel calcolo degli ordini dei costituenti maggiori riassunto alla tavola 3. tutte queste frasi sono state ridotte a parallele frasi sem-

12 Il fenomeno dell'inversione di sequenza fra soggetto e oggetto diviene anche più evidente di quanto appaia dai dati numerici se si tiene conto del fatto che alcuni ordini con $\mathrm{S}>\mathrm{O}$ sono dovuti in realtà a soggetti peculiari, dei pronomi relativi, la cui posizione iniziale di frase è obbligatoria: cfr. ess. (19) $[$... In maniera un po'brutale, che non mi convince, $A M E$ in primo luogo (ordine SVO), e (29) $[. .]$. che a loro disturba (ordine SOV).

13 E' indubbio tuttavia che lo statuto rematico che si percepisce nei soggetti postverbali è anche effetto della loro stessa posizione; nei casi in cui non c'è una chiara differenza di 'peso' sintattico, su questo punto la riflessione rischia di essere circolare.

$14 \mathrm{Cfr}$. anche l'es. 37. Invece in (90) ho pensato quanto avrebbe stancato $A$ ME quel viaggio l'ordine col $V$ all'inizio è dovuto allo statuto della frase subordinata, che è una interrogativa indiretta con fuoco su un àverbiale: è normale in questo caso che i costituenti nominali seguano il verbo; l'ordine relativo OS è invece da riportare al diverso 'peso' di O e S, e rispetta la regola generale che stiamo commentando. 
plici, la (91) come OSV, le altre come OVS. ${ }^{15}$ In ogni caso, in queste frasi il soggetto è chiaramente rematico.

Nei casi in cui sia il soggetto che l'oggetto precedono il verbo (12 occorrenze con OSV, 4 con SOV), troviamo spesso soggetti che sono topic di discorso, hanno cioè una componente anaforica: per es. in (36), con ordine SOV, la valeriana a me mi eccita in un modo-, la valeriana riprende l'argomento del dialogo in corso; in (33), con ordine OSV, Ai bambini di certo l'idea non disturba, l'idea rinvia al «vivere in una società multirazziale» citato prima (altri esempi analoghi: 31, 53). Altre volte il soggetto è deittico (cfr. 8, al gatto io lo coccolo...), oppure ha una posizione preverbale obbligata perché fuoco di interrogativa (es. 17, e a me chi mi consola?) o pronome relativo (es. $29,[\ldots]$ che a loro disturba). Nell'insieme, tenendo conto anche dei casi di ordine SVO, i soggetti preverbali sono chiaramente più 'leggeri' di quelli postverbali (per es., dei 5 casi con ordine SVO, quattro hanno soggetti pronominali deittici - ess. $1,14,47,83-$, uno ha come soggetto un pronome relativo es. 19).

Per completare il quadro dell'acc. prep. come topic frasale che va chiaramente emergendo da tutti i dati di sintassi, dobbiamo citare ancora una caratteristica frequente nel corpus, la presenza della negazione fra l'oggetto preposto e il resto della frase: si vedano esempi come (20) a me non convince, (25) se a te non ti disturba, $\mathrm{e}$ molti altri. Vi sono 17 casi con $O N e g V$, che diventano 18 se vi si include anche l'es. 79 con nessuno a soggetto: a me nessuno mi protegge; in altri 4 casi anche il soggetto precede la negazione, come in 21, A ME quelle carte non convincono. L'acc. prep. dunque tende a cadere fuori dallo scope della negazione, secondo il comportamento tipico degli elementi topicali, che sono presupposti e come tali non negati/negabili. ${ }^{16}$ A lato, notiamo anche che dal punto di vista meramente lineare la negazione, assieme alla sequenza OSV (e, al massimo grado, alle strutture pseudoscisse o scisse con acc. prep. all'inizio), allontana dal verbo l'oggetto preposto, accentuandone le caratteristiche di topic frasale.

\section{VERBI REGGENTI}

Si è già fatto cenno sopra all'importanza dei verbi reggenti, ed in particolare ai verbi cosiddetti 'psicologici', per la sintassi dell'acc. prep.; vediamo ora di concretizzare questo punto con dati relativi alla posizione dell'acc. prep. e alla presenza o assenza del clitico.

15 Valutando come soggetto non il costituente posposto bensì la formula introduttiva quello (che), i cinque casi con acc. prep. al primo posto sarebbero da calcolare come OSV, e l'es. (12) citato invece come SOV: cambierebbe il rapporto fra ordini OVS e OSV; ma mi pare che non vi siano dubbi sul fatto che nelle pseudoscisse il vero soggetto è l'ultimo costituente della struttura.

16 Vi sono però anche acc. prep. postverbali in frasi negative (ess. 18 , non mi convince $A M E ; 100$, non t'ho visto a te; nonché $19,27,98)$, in cui l'oggetto cade nello scope della negazione. 
Sulla base delle osservazioni di Benincà (1986, e in Renzi 1988), ${ }^{17}$ nonché di indizi emersi in lavori precedenti (Berretta 1989b e 1990b), i verbi reggenti gli acc. prep. del nostro corpus sono stati divisi in tre gruppi: verbi psicologici (affascinare, attrarre, colpire «impressionare», ecc.), ${ }^{18}$ complessi verbali causativi (infiniti retti da fare o lasciare) e altri verbi (accompagnare, aspettare, e così via sino a vedere). Le differenza potenzialmente significativa è data dal fatto che gli oggetti di verbi psicologici e di complessi verbali causativi non sono 'veri' complementi oggetti: i primi per motivi semantici, in quanto sono gli esperienti di stati ed eventi mentali, ed assomigliano in questo a soggetti (per esempio, l'oggetto di spaventare ha un ruolo semantico analogo al soggetto di temere); i secondi per motivi sintattici, in quanto l'oggetto dell'intero complesso causativo è in realtà il soggetto del verbo incassato. Ovviamente anche con gli altri verbi gli acc. prep. non costituiscono oggetti prototipici, ma se ne allontanano in grado relativamente minore.

I verbi individuati sono elencati qui di seguito, in ordine alfabetico e con l'indicazione di occorrenze superiori a una (per gli esempi si rinvia sempre all'appendice). Nei complessi verbali costituiti da un modale ed un infinito si è indicato, ovviamente, il verbo retto (ess. $10,88,99$ ). Si noti che è stato necessario, in almeno un caso, categorizzare in modo diverso lo stesso verbo a seconda del contesto: mettere (di buon umore), psicologico, vs. mettere (in galera), non psicologico. ${ }^{19}$

(i) Verbi psicologici (che costruiscono l'esperiente come oggetto diretto): affascinare, attrarre (2), colpire «impressionare» (4), confortare, consolare, convincere (4), deludere (2), disturbare (9), divertire (2), eccitare, entusiasmare, incantare, ingannare, innervosire, invitare «attirare», irritare, mettere (di buon umore), persuadere, preoccupare (5), rattristare, spaventare.

(ii) Complessi verbali con fare e lasciare causativi: fare + infinito (6: $f$. dormire, $f$. ingrassare, $f$. morir dal ridere, $f$. ridere, $f$. piangere, $f$. sentir male), lasciare + infinito (2: $l$. salire, $l$. stare).

17 In realtà l'Autrice non tratta gli oggetti di verbi psicologici marcati con $a$ come veri acc. prep., poiché ritiene che la comparsa della preposizione sia dovuta alle proprietà specifiche di questi verbi, il cui oggetto è in realtà l'esperiente, e quindi tende ad emergere in superficie con una marca di dativo. Per una discussione di questo punto rinvio a Berretta $1989 \mathrm{~b}$, pp. 24-31, in cui. ho cercato di mostrare come gli oggetti marcati con $a$ (retti da verbi sia psicologici che non) sono acc. prep. e non dativi. L'argomento più forte in questo senso è, credo ancora, il confronto tipologico generale e specifico con le altre lingue romanze. $\mathbb{E}$ ' vero comunque che acc. prep. e (almeno alcuni) dativi hanno in comune l'inclinazione allo statuto di tema (sui dativi cfr. Salvi 1986), il che va riportato a mio avviso a comuni tratti di animatezza e definitezza dei nominali che emergono nei due ruoli sintattici.

18 Sui verbi psicologici in genere (quelli che qui ci interessano, che hanno l'esperiente costruito come oggetto diretto, ma anche quelli che lo costruiscono come soggetto, per es. amare, o come dativo, per es. piacere) e sul loro comportamento sintattico, visto in chiave di grammatica generativa, si veda Belletti e Rizzi 1988.

19 Non mancano, ovviamente, casi dubbi: per esempio eccitare, visto nel suo contesto (es. 36), non sarebbe forse da considerare 'psicologico'; alcuni fare causativi (fare ridere e far dormire) viceversa costituiscono con il verbo retto dei complessi molto simili a verbi psicologici. Anche la generica categoria dei verbi 'altri' avrebbe forse potuto essere ulteriormente suddivisa, per esempio evidenziando i verbi che includono il significato di «parlare a qualcuno» (chiamare, ringraziare, salutare), i percettivi (vedere), e forse altri: per un simile lavoro sarebbe però necessario un corpus più ampio di quello qui esaminato. 
(iii) Altri verbi: accompagnare, aspettare (2), chiamare, coccolare, conoscere (3), danneggiare, fermare, fregare, fucilare, graffiare, incastrare, informare, incolpare, lasciare (in pace), mandare, mettere (in galera), pagare (5), picchiare, portare (4), prendere, proteggere, pungere, ringraziare (3), rovinare, salutare, seppellire, sposare, stancare, stendere, svegliare (2), temere, toccare «coinvolgere», trattare (male), vedere (4).

Le occorrenze totali sono così distribuite: 42 verbi psicologici, 8 complessi causativi, e 50 altri verbi. Scorporando queste cifre a seconda della posizione rispetto al verbo ed alla presenza o assenza del clitico si hanno cifre ovviamente piccole, ma con un andamento significativo (tavola 4.) almeno per i verbi psicologici e gli 'altri verbi'; i numeri assoluti relativi a fare e lasciare causativi sono invece così ridotti da richiedere cautela in tutte le interpretazioni.

Tav. 4. Verbi reggenti

\begin{tabular}{lrcrr} 
& v. psic. & $\begin{array}{c}\text { complessi v. } \\
\text { causativi }\end{array}$ & altri & Tot. \\
indecidibili & 2 & 0 & 6 & 8 \\
prev. con clit. & 8 & 2 & 26 & 36 \\
prev. senza clit. & 29 & 5 & 4 & 38 \\
post. con clit. & 2 & 1 & 12 & 15 \\
post. senza clit. & 1 & 0 & 2 & 3 \\
Tot. & 42 & 8 & 50 & 100 \\
\hline
\end{tabular}

Come ci aspettavamo in base alla natura degli oggetti e al rapporto tra verbi e oggetti stessi, i verbi psicologici e le perifrasi causative hanno una preferenza più netta per la posizione preverbale dell'acc. prep.: gli psicologici hanno su 40 occorrenze 'decidibili' 37 posizioni preverbali $(92,5 \%)$, i causativi 7 su $8(87,5 \%$, con il caveat detto sopra), gli altri verbi 30 su $44(68,2 \%)$. Gli acc.prep. retti da verbi psicologici e perifrasi causative sono, in altri termini, più spesso topic di frase di quanto lo siano gli acc. prep. retti da altri verbi.

La seconda e forse più importante differenza è data dalla maggiore inclinazione degli acc. prep. retti da psicologici e causativi a non avere ripresa clitica. Il dato è evidente nella tabella, e si ricava sia paragonando le sole occorrenze preverbali, dove gli psicologici hanno 29 su 37 casi senza ripresa, i causativi 5 su 7 e gli altri verbi solo 4 su 30, sia tenendo conto di tutte le occorrenze decidibili, sia pre- che postverbali: in questo caso abbiamo $30 / 40(75 \%)$ per i verbi psicologici, $5 / 8(62,5 \%)$ per i causativi e $6 / 44(13,6 \%)$ per gli altri verbi. I casi tipici dei tre gruppi di verbi sono quindi riassumibili rispettivamente nei tipi a me non convince (es. 20), in posizione preverbale e senza clitico, a me fanno piangere (es. 42), con le medesime caratteristiche, e a me mi conoscono (es. 16), sempre in posizione preverbale ma con clitico. 
Ciò significa a nostro avviso due cose. Anzitutto, che gli acc. prep. retti da psicologici e causativi sono maggiormente integrati nella frase di quanto avvenga con altri verbi: benché per natura e posizione siano massimamente topicali, di preferenza non vanno a costituire dislocazioni a sinistra. In secondo luogo, la scarsità di strutture con dislocazione - che sono tipicamente colloquiali - è indizio del carattere sociolinguisticamente non marcato dell'acc. prep. con verbi psicologici e complessi causativi (già osservato da Benincà per i verbi psicologici), che infatti occorre più spesso in varietà sorvegliate. Si confrontino a me non convince e $a$ me non mi convince, a me fanno piangere e a me mi fanno piangere: l'inserzione del clitico è possibile, ma abbassa di registro le medesime frasi (viceversa a me conoscono è, rispetto a a me mi conoscono, non più alto di registro ma semplicemente 'strano', come l'esempio reale 59, a me hanno mandato giù per sbaglio).

Un'ulteriore questione che occorrerebbe affrontare, ma che esce dai confini e dalla metodologia di questo lavoro, è l'obbligatorietà della preposizione: in molti dei contesti preverbali, almeno nella competenza di chi scrive, la forma senza $a$ è impossibile (*/??me non convince $\mathrm{e} /$ ?? me fanno piangere $)$, o diviene accettabile con intonazione contrastiva: $M E$ non convince, $M E$ fanno piangere (non mia sorella), $\mathrm{e}$ simili. ${ }^{20}$ Migliore, ma nettamente più bassa di registro, è la soluzione senza preposizione ma con ripresa clitica, del tipo me mi conoscono, te t'ho visto, e simili, in cui l'oggetto viene trattato come tema libero. ${ }^{21}$ In contesti postverbali, che abbiamo detto essere più marcati, invece la preposizione può sempre essere omessa: non covince me, ho visto te ecc. (si noti che è qui nettamente migliore la versione senza clitico: si torna ad un normale ordine $\mathrm{VO}$, con $\mathrm{O}$ enfatico perché reso col pronome tonico).

\section{CONCLUSIONI}

L'esame dei dati che abbiamo compiuto nei paragrafi precedenti non ha portato novità sostanziali rispetto a quanto già si sapeva sull'acc. prep. italiano: ci ha però consentito di concretizzare e precisare riflessioni ed ipotesi avanzate sia da chi scrive in lavori precedenti sia da altri autori.

20 In realtà più variabili influenzano l'(in)accettabilità di oggetti preverbali non marcati: oltre alla posizione pre-o postverbale e alla presenza/assenza del clitico, certamente il tipo di verbo reggente, poiché verbi psicologici e causativi preferiscono $a$ (Beninicà 1986); a mio avviso poi anche la distanza dal verbo vi ha un ruolo. Così per es. noi c'ha svegliato il telefono (cfr. 92, a noi c'ha svegliato il telefono), con adiacenza oggetto-verbo, mi suona migliore di me è 'sto periodo di caldo che mi ha steso (cfr. 91, a me è 'sto periodo di caldo che mi ha steso), in cui l'oggetto non marcato da preposizione risulta separato dal verbo, in posizione di tema libero. Ma in entrambi i casi siamo in varietà marcatamente colloquiali, nelle quali non è facile dare giudizi metalinguistici (e si veda, alla nota che segue, un controesempio).

21 Ho un unico esempio (scritto) di contesto che richiederebbe acc. prep. e invece non l'ha, con un fare causativo: Me, le cose torride mi fanno ridere (intervista a S. Corbucci, in «L'Espresso Più» dic. 89, p. 79): è possibile che si tratti di ipercorrettismo. 
Il quadro teorico generale in cui si inscrivono i sistemi a marcatura differenziale dell'oggetto ne è uscito ulteriormente confermato anche per quanto riguarda la nostra lingua. Partendo dal principio che il soggetto tenda ad essere un elemento tematico, definito e con referente animato, e viceversa l'oggetto tenda ad essere rematico, indefinito e con referente inanimato (Givón 1976, Keenan 1976, Comrie 1979, e altri), l'acc. prep. risulta una marca esplicita di caso che emerge quando l'oggetto è anomalo in quanto presenta le caratteristiche tipiche del soggetto. Da questo punto di vista l'italiano differisce da altre lingue romanze per l'ambito ristretto cui confina l'acc. prep., ma non per la natura del fenomeno.

Il carattere anomalo degli oggetti che occorrono marcati con preposizione è emerso sia direttamente, nelle loro caratteristiche referenziali inerenti (referenti umani, singolari), di definitezza e specificità (netta prevalenza dei pronomi deittici) e di carattere tematico (preferenza per la posizione preverbale), sia indirettamente, in caratteristiche speculari dei soggetti, che abbiamo visto essere spesso rematici. Nei casi in cui il soggetto è espresso è infatti emersa una preferenza per la sua posposizione rispetto all'oggetto, il che come s'è detto sarebbe contrario ad un universale tendenziale che invece in questo caso, per la distribuzione anomala delle caratteristiche dei due elementi, risulta rispettato.

La natura di tema o topic frasale degli acc. prep., o almeno di buona parte di essi, induce a pensare che la preposizione, mentre esplicita il ruolo sintattico dell'elemento marcato, ne assicuri anche l'integrazione nella struttura frasale, evitando che risulti un tema libero. Da questo punto di vista la preposizione non è, come alcuni hanno sostenuto, una marca di topic, bensì al contrario un segnale del fatto che il topic non è sganciato dalla frase ma ne fa parte, con un preciso ruolo sintattico. Il fenomeno è particolarmente evidente nei casi in cui l'acc. prep. compare all'inizio della frase ed è separato dal verbo da altro materiale, talché l'interpretazione quale freies Thema sarebbe, senza marca, ovvia.

Un punto delicato, ma particolarmente utile per il lettore non nativo, è il giudizio sul grado di accettabilità dell'acc. prep. in italiano. Come si è detto più volte, abbiamo lavorato su dati non marcati in diatopia, ma per lo più di registro colloquiale o scritto non formale. All'interno di questa caratterizzazione sociolinguistica, che vale in genere per gli ambiti d'uso dell'acc. prep. in italiano, abbiamo visto che l'acc. prep. è non solo preferibile, ma addirittura obbligatorio in alcuni contesti, in particolare in posizione preverbale con verbi reggenti psicologici o complessi verbali retti da causativi. In questi stessi contesti è preferibile l'assenza di ripresa clitica, ovvero la totale integrazione dell'oggetto marcato nella frase (per es. a me attrae, migliore di a me $m$ 'attrae). Va notato anche che in questi intorni talvolta non è possibile, o meglio è inelegante, la trasformazione della frase in passiva, che altrimenti risolve egregiamente il compito di portare a tema un oggetto: io sono conosciuto/a è il parallelo formale, ed elegante, di a me mi conoscono, mentre io sono consolato/a o io sono convinto/a sono ineleganti quali passivi per a me consola e a me convince (sono, invece, normali come forme risultative, con copula più aggettivo: ma 
l'accezione è diversa), e infine del tutto impossibile è la trasformazione passiva con i causativi (**io sono fatto ridere o simili). ${ }^{22}$

In conclusione, l'accusativo preposizionale, con le preferenze che sono state descritte relativamente al tipo di oggetti, al loro carattere tematico e ai verbi reggen$\mathrm{ti}$, è da considerare un fenomeno morfosintattico normale, anche se poco frequente in termini numerici, nell'italiano dell'uso medio. Questo fenomeno, con altri tipici della stessa varietà (dislocazioni a sinistra e a destra, ordini VS, ecc.), mostra che la nostra lingua è più vicina al tipo romanzo dominante di quanto sembri esserlo l'italiano standard letterario.

\section{APPENDICE ${ }^{23}$}

accompagnare

(1) è la malinconia di dover dirsi ciao + allora scatta l'idea 'me + io ti accompagno a casa te e te tu $m$ 'accompagni anche $a m e$ ' [intervista a cantante bolognese, probabilmente semic., alla radio: dal corpus inedito di italiano parlato di E. Mara (Graz)]

affascinare

(2) a me è quella che affascina di più [immagine di una casa]

aspettare

(3) Sferragliavano i tram gremiti di ragazzi, a loro le aspettava Adone in doppio petto blu. [R. Loy, La bicicletta, Torino $1989^{2}$, p. 45]

(4) allora, vi aspetto più tardi + a tutt'e due. Ciao!

attrarre

(5) A me quello che attrae in un uomo è l'aria emblematica, lo sguardo sensibile [intervista a M. Ripa di Meana, in «L'Espresso Più» nov. 1988]

(6) a me quello che attrae di più è l'idea [...]

chiamare

(7) a chi hai chiamato, adesso? [ = «a chi hai telefonato?»]

coccolare

(8) al gatto io lo coccolo più spesso che i miei figli [probabilmente semic.]

colpire

(9) A me colpì moltissimo il fatto che anni fa si scoprisse che ai vertici della Guardia di Finanza avevano fatto il nido personaggi in collusione col contrabbando di benzina. [E. Scalfari, in «Il Venerdì di Repubblica» 10. 2. 89]

22 La forma passiva con l'ausiliare essere sembra invece normale con alcuni verbi psicologi, per es. sono attratto/a da..., sono colpito/a da..., ecc.; mi pare comunque impossibile l'uso di venire come ausiliare del passivo per questi verbi, il che va correlato alla loro Aktionsart di stativi.

23 Sono qui riportate tutte le 100 occorrenze esaminate, ordinate alfabeticamente in base al verbo reggente. Ove non diversamente specificato, va inteso che l'esempio è trat to da conversazione informale, e che il parlante è un adulto colto o molto colto, piemontese o lombardo. Fonti diverse sono invece sempre esplicitate, con le abbreviazioni che seguono. Per la situazione: semif. per parlato semiformale o formale (esempi tratti da interventi in riunioni di consigli accademici, congressi scientifici, e simili). Per parlanti: stud. univ. per studente/essa universitario/a; semic. per parlante semicolto, con licenza di scuola media inferiore. 
(10) Ad un linguista possono colpire particolarmente [...] frasi del seguente tenore [Giovan Battista Pellegrini, Minoranze e culture regionali, Padova 1986, p. 6]

(11) a me ha colpito un aspetto in particolare [semif.]

(12) quello che a me colpisce è [...]

confortare

(13) ti conforta? a me, no, non mi conforta [semif.]

conoscere

(14) io $t i$ conosco, a te! [uomo probabilmente semic., parla scherzosamente con una bambina piccola]

(15) beh, non è che ci conoscano tanto + a te magari si

(16) perché a me mi conoscono

consolare

(17) e $a$ me chi mi consola?

convincere

(18) non $m i$ convince $A M E$

(19) [...] in maniera un po' brutale, che non $m i$ convince, $A M E$ in primo luogo [semif.]

(20) io ve lo dò + però, $a$ me non convince [semif.]

(21) A ME quelle carte non convincono

danneggiare

(22) a me danneggia tantissimo quello della settimana dopo [sciopero ferroviario]

\section{deludere}

(23) (24) sai che $a m e m$ 'ha deluso la Bretagna? [...] ma- $a$ me ha deluso moltissimo

disturbare

(25) se $a$ te non $t i$ disturba

(26) A: vi disturbo se faccio fotocopie mentre voi parlate? B: a me, no

(27) non disturba a noi [semif.]

(28) a me non disturba così tanto

(29) [...] che a loro disturba [loro è riferito ai gatti di casa]

(30) a me non disturba se viene nero [il carciofo]

(31) A: [il movimento della freccia del mouse sullo schermo] mi disturba B: anche a me quello disturba

(32) Io sento un piccolo rumore di fondo, non so se lo sentite anche voi $+a$ me comunque non $m i$ disturba [telefonata di ascoltatore, RDue 13. 3. 91]

(33) Prepariamoci a vivere in una società multirazziale. [...] Ai bambini di certo l'idea non disturba ['Pubblicità Progresso' in «L'Espresso» 14. 4. 91]

divertire

(34) sì $a$ me diverte tutto quello che ha a che vedere col [...] [semif.; «Parola mia», Rai 1]

(35) a me diverte fare lezione

eccitare

(36) la valeriana a me mi eccita in un modo-

entusiasmare

(37) a te non entusiasmano, le carote cotte

fare + infinito

(38) a me il sonnifero ha fatto dormir bene 
(39) a me fa ingrassare [rifiutando un dolce offerto]

(40) a me quel che ha fatto morir dal ridere è stato ricevere, sotto tre forme diverse, gli auguri di Natale

(41) ma a voi fa ridere uno che dice [...] [G. Bocca in «La Repubblica» 8. 9. 89]

(42) a me fanno piangere [film commoventi] [giovane donna di ceto medio]

(43) Del resto, $a$ me, la realtà di un Bukowski $m i$ fa sentir male [intervista a O. Muti, in «Il Venerdi di Repubblica»' 13. 10. 89]

fermare

(44) a me $m$ hanno fermato, la polizia [semic.]

fregare

(45) A: ed è li che ti frego B: $A C H I$ ? [ribatte ironicamente]

fucilare

(46) che cos'è, $m i$ hanno fucilato $A M E$ ? [pezzo teatrale, RTre 9. 3. 91]

graffiare

(47) ti piacerebbe se io $t i$ graffiassi cosi $A T E$ ? [rivolto al gatto di casa]

incantare

(48) [...] un posto bellissimo, a me mi incanta [semic.]

incastrare

(49) perché a me $m$ 'incastra, quello lì

informare

(50) ma questi a noi non c'hanno informato per niente [semif.]

incolpare

(51) professoressa, $m$ 'incolpano a me! [ragazzi di una prima media di Torino - cfr. (69) e (70)]

ingannare

(52) a me non mi si inganna

innervosire

(53) a me questa storia innervosisce

invitare

(54) a me invita [ = «attira», un cibo] [semic.]

irritare

(55) a me alcune persone $m i$ irritano

lasciare (in pace)

(56) Certo, facciamoli lavorare [...] Così a noi bianchi ci lasciano in pace, qui in piazza Duomo [intervista a venditore ambulante, in "La Repubblica» 28. 5. 89]

lasciare + infinito

(57) ma intanto lasciateci salire a noi [sul treno]

(58) finora a noi ci lasciavano stare, le bande mafiose si sparavano tra di loro [intervista a poliziotto moscovita, in «La Repubblica» 21. 10. 90]

mandare

(59) A: ci riincontriamo B: $a$ me hanno mandato giù per sbaglio 
mettere (di buon umore)

(60) tutte le faccende natalizie $a$ me non metton mai di buon umore

mettere (in galera)

(61) Se questa guerra continua a noi nordafricani $c i$ metteranno tutti in galera [intervista a nordafricano, in «La Repubblica» 30. 1. 91]

pagare

(62) A: non mi hanno mai pagato $\mathrm{B}$ : ah, questo neanche $a$ me

(63) tanto a Lei la pagano

(64) a me mi hanno pagato

(65) (66) a te ti paga e a me pure $m i$ paga [da sceneggiato televisivo, in «Mercurio» suppl. a «La Repubblica» 21. 7. 90]

persuadere

(67) a me non persuade

picchiare

(68) A: [vostro padre] vi picchiava- B: ci picchiava a noi?! no- [semic.]

portare

(69) e perché a noi non ci portate [in gita]? [come 51]

(70) un'altra volta ci portate anche $a$ noi [come 51 e 69]

(71) A: mi ricordo, quando andavamo a scuola c'era la festa degli alberi, si andava a piantare una pianta- B: a noi $c i$ portavano solo in chiesa

(72) Non escludo che in questo giudizio vi sia un po' di invidia, quando leggo che Oriana «if WIA or KIA», se ferita o uccisa, avrebbe voluto far tornare il corpo, sanguinante o esamine, all'ambasciata di Saigon o addirittura alla Casa Bianca. $A$ noi comuni mortali, ci portano alle Usl. ['Lettera aperta' di A. Barbato, in «L'Espresso» 5. 8. 90]

prendere

(73) Mica sono razzista, solo che da quando ci sono loro a noi sul lavoro non ci prendono più [intervista a trentenne fiorentina, in «L'Espresso» 11. 11. 84]

preoccupare

(74) a me non preoccupa

(75) (76) $A$ me preoccupa Torino: è una città difficile [...] $A$ Cerami invece preoccupa Viterbo [intervista a R. Benigni, "La Stampa» 9. 10. 88]

(77) Anche a me preoccupa molto il fatto da Lei denunciato [E. Scalfari in «Il Venerdi di Repubblica» 4. 5. 90]

(78) a me già preoccupa questo trionfo [...]

proteggere

(79) Interrompe Donat Cattin: « $A$ me nessuno $m i$ protegge» [«La Repubblica» 10. 2. 89] pungere

(80) " $a$ te non $t i$ pungono mai, devi avere il sangue amaro" dice lei [R. Loy, La bicicletta, Torino, $1989^{2}$, pp. 100-101]

rattristare

(81) per fortuna a me la dieta non rattrista

ringraziare

(82) A: perfetto, La ringrazio B: a Lei, A.; arrivederci. 
(83) A: grazie! B: io ringrazio $A L E I$ ! buongiorno! [anziano sarto di origine emiliana; semic.]

(84) A: grazie B: La ringrazio a Lei [semic.]

rovinare

(85) Però a me mi ha rovinato la Musa [titolo di articolo, in «Mercurio», suppl. de «La Repubblica» 21. 10. 89]

salutare

(86) ti saluta Renzo [ad altro interlocutore] anche a te

seppellire

(87) $a$ me $m i$ avete seppellito [di regali]

sposare

(88) Lui ama un'altra [...] la vuole sposare: $a$ me non $m i$ ha mai voluto sposare [dal film "Africana", in «II Venerdi di Repubblica» 6. 4. 90]

spaventare

(89) La perestrojka [...] $A$ me spaventa perché temo che tutta questa smania di produrre finisca col distruggere le nostre risorse [intervista a sceneggiatrice sovietica, in «La Repubblica» 1. 11.89]

stancare

(90) ho pensato quanto avrebbe stancato $A M E$ quel viaggio

stendere

(91) a me è 'sto periodo di caldo che $m i$ ha steso

svegliare

(92) a noi c'ha svegliato il telefono, verso le quattro

(93) tu hai dormito tutta la notte? a me ha svegliato la sveglia

temere

(94) a me non mi teme

toccare

(95) non so se anche tu hai questa grana dei lettori + no, a te non ti tocca così da vicino trattare (male)

(96) A: non ti ho mica trattato male... B: io $\mathrm{t} / \mathrm{a}$ te sì però

vedere

(97) a me non $m i$ vedi fare queste cose

(98) te ne liberi per un anno non lo vedi più a quello [un ragazzo] [stud. univ.]

(99) vorrei vederti a te fare otto ore di allenamento al giorno

(100) ma non $t$ 'ho visto a te [stud. univ.]

\section{BIBLIOGRAFIA}

Belletti, Adriana e Rizzi, Luigi, Psych-verbs and 0-theory, in «Natural Language and Linguistic Theory» 6 (1988), pp. 291-352.

Benincà, Paola, Il lato sinistro della frase italiana, in «The ATI Journal» 47 (1986), pp. $57-85$. 
Berretta, Monica, Tracce di coniugazione oggettiva in italiano, in: Foresti, Fabio, Rizzi, Elena e Benedini, Paola (a cura di), L'italiano fra le lingue romanze, Atti del XX Congresso della S.L.I. (Bologna, 25-27 settembre 1986), Roma (Bulzoni) 1989, pp. 125-150 [= 1989a].

Berretta, Monica, Sulla presenza dell'accusativo preposizionale in italiano settentrionale: note tipologiche, in «Vox Romanica» 48 (1989), pp. 13-37 [ = 1989b].

Berretta, Monica, «E a me chi mi consola?» [Sull'accusativo preposizionale in italiano], in "Italiano e Oltre» $5(1990) / 1,31-35$ [ = 1990a].

Berretta, Monica, Sull'accusativo preposizionale in italiano, in Berretta, Monica, Molinelli, Piera e Valentini, Ada (a cura di), Parallela 4. Morfologia / Morphologie, Tübingen (Narr) 1990, pp. 179-189 [ = 1990b].

Berretta, Monica, Il parlato italiano contemporaneo, in: Serianni, Luca e Trifone, Pietro (a cura di), Storia della Lingua Italiana, vol. I., Torino (Einaudi), in stampa.

Berruto, Gaetano, Sociolinguistica dell'italiano contemporaneo, Roma (La Nuova Italia Scientifica) 1987.

Bossong, Georg, Empirische Universalienforschung. Differentielle Objektmarkierung in den neuiranischen Sprachen, Tübingen (Narr) 1985.

Bossong, Georg, On objects in language and the objects of linguistics [recensione a Plank 1984], in «Lingua» 69 (1986), pp. 139-164.

Bossong, Georg, Differential object marking in Romance and beyond, in: Kibbee, Douglas e Wanner, Dieter (eds.), New analyses in Romance Linguistics, Papers from the Linguistic Symposium on Romance Linguistics 18 (University of Illinois, Urbana-Champaign, 1988), Amsterdam (Benjamins), in stampa.

Comrie, Bernard, Definite and animate direct objects: a natural class, in "Linguistica Silesiana» 3 (1979), pp. 13-21.

Diez, Friedrich, Grammatik der romanischen Sprachen, Bonn (Weber) 1836/43, $1882^{5}$.

Elia, Annibale, Note su una sintassi italiana tra dialetto e lingua. La costruzione ' $N V$ a $N$ ' come realizzazione regionale meridionale dell'itatiano standard ' $N V N$ ', in: Albano Leoni, Federico (a cura di), I dialetti e le lingue delle minoranze di fronte all'italiano, Atti dell'XI Congresso della S.L.I. (Cagliari, 27-30 maggio 1977), Roma (Bulzoni) 1980, pp. 83-98.

Givón, Talmy, Topic, pronoun and grammatical agreement, in Li 1976, pp. 149188.

Greenberg, Joseph H., Some universals of grammar with particular reference to the order of meaningful elements, in: Greenberg, J. H. (ed.), Universals of Language, Cambridge (MIT Press) 1963, 1966², pp. 73-113; trad. it. in: Ramat, Paolo (a cura di), La tipologia linguistica, Bologna (Il Mulino) 1976, pp. $115-154$.

Keenan, Edward L., Towards a universal definitions of "subject", in Li 1976, pp. 303-333.

Körner, Karl-Hermann, Korrelative Sprachtypologie. Die zwei Typen romanischer Syntax, Stuttgart (Steiner) 1987. 
Lazard, Gilbert, Actance variation and categories of the object, in Plank 1984, pp. 269-292.

Leone, Alfonso, L'italiano regionale in Sicilia, Bologna (Il Mulino) 1982.

Li, Charles N. (ed.), Subject and Topic, New York (Academic Press) 1976.

Loi Corvetto, Ines, L'italiano regionale di Sardegna, Bologna (Zanichelli) 1983.

Moravcsik, Edith A., On the case marking of objects, in: Greenberg, Joseph $\mathrm{H}$. (ed.), Universals of Human Language, Vol. IV. Syntax, Stanford (Stanford University Press) 1978, pp. 249-289.

Nocentini, Alberto, Sulla genesi dell'oggetto preposizionale nelle lingue romanze, in: Studi Linguistici e Filologici per Carlo Alberto Mastrelli, Pisa (Pacini), 1985, pp. 299-311.

Nocentini, Alberto, Oggetto marcato vs. oggetto non-marcato: stato ed evoluzione di una categoria nell'area euro-asiatica, in: Soravia, Giulio et alii (a cura di), L'Europa linguistica: contatti, contrasti, affinità di lingue, Atti del XXI Congresso della S.L.I. (Catania, 10-12 settembre 1987), Roma (Bulzoni), in stampa.

Plank, Frans (ed.), Objects. Towards a Theory of Grammatical Relations, New York (Academic Press) 1984.

Renzi, Lorenzo (a cura di), Grande grammatica italiana di consultazione, Vol. I, La frase. I sintagmi nominale e preposizionale, Bologna (Il Mulino) 1988.

Rohlfs, Gerhard, Historische Grammatik der italienischen Sprache und ihrer Mundarten. III. Syntax und Wortbildung, Bern (Francke) 1954; trad. it. Grammatica storica della lingua italiana e dei suoi dialetti. Vol. III. Sintassi e formazione delle parole. Torino (Einaudi) 1969.

Rohlfs, Gerhard, Autour de l'accusatif prépositionnel dans les langues romanes, in «Revue de Linguistique Romane» 35 (1971), pp. 312-334.

Rohlfs, Gerhard, Panorama de l'accusatif prépositionnel en Italie, in «Studii şi cercetări linguistice» 24/5 (1973), pp. 617-621.

Sabatini, Francesco, L'«italiano dell'uso medio»: una realtà tra le varietà linguistiche italiane, in: Holtus, Günter e Radtke, Edgar (Hrsg.), Gesprochenes Italienisch in Geschichte und Gegenwart, Tübingen (Narr) 1985, p. 154-184.

Salvi, Giampaolo, Asimmetrie soggetto/tema in italiano, in: Stammerjohann, Harro (ed.), Tema-Rema in Italiano, Tübingen (Narr) 1986, pp. 37-53.

Serianni, Luca [con la collaborazione di A. Castelvecchi], Grammatica italiana. Italiano comune e lingua letteraria, Torino (UTET) 1988.

Silverstein, Michael, Hierarchy of features and ergativity, in: Dixon, R. M. W. (ed.), Grammatical Categories in Australian Languages, Canberra (Australian Institute of Aboriginal Studies) 1976, pp. 112-171.

Tekavčić, Pavao, Grammatica storica della lingua italiana, 3 voll., Bologna (Il Mulino) 1972, $1980^{2}$.

Terracini, Lore, Alcuni usi sintattici nel «Libro de buen amon», tesi di laurea non pubblicata, Università di Torino, 1950.

Zamboni, Alberto, Postille alla discussione sull'accusativo preposizionale, Relazione al XIX Congresso Internazionale di Linguistica e Filologia Romanza (Santiago de Compostela, 4-9 settembre 1989), in stampa nei relativi Atti. 


\section{Povzetek}

\section{OPOMBE K SKLADNJI PREDLOŽNEGA TOŽILNIKA V ITALIJANŠČINI}

V italijanščini srednjega registra, tako kot v nekaterih drugih romanskih jezikih, je znan predložni tožilnik, se pravi, razločevalno označevanje predmeta, glede na to, ali gre za osebo: predmet v tožilniku, zlasti še, če gre za zaimek, je označen s predlogom $a$. Na osnovi stotih primerov je opisana skladnja predložnega tožilnika, kot jo najdemo v italijanšcini srednjega registra, ne visokega. Posebej je upoštevana in pretresana pozicija tega stavčnega člena glede na povedek: predmet stoji v glavnem pred povedkom, kar ustreza tematski vsebini predmeta, stoji pa večinoma tudi pred osebkom. Analizira se nadalje pojavljanje povzemalnega nenaglašenega osebnega zaimka in pretresa se pomenska vrednost glagolov, od katerih je predmet odvisen.

Ugotavlja se, da glagoli izrazite psihične vsebine in vzročni glagoli pogosteje zahtevajo rabo objekta pred glagolom brez povzemalne naslonke, prim.: a me non convince 'me ne prepričuje'; a me fanno piangere 'spravljajo me v jok', kar kaže na večje zlitje objekta v stavek; pri drugih glagolih je mogoče zaslediti naslonke pred glagolom, ali tudi za njim: a me mi conoscono 'poznajo me'; t'ho visto a te 'videl sem te'. 